\title{
Application of a completely organic and bio- degradable sugar-based insulating coating to vine shoots, to prevent late frost damages
}

\author{
Alessia Di Giuseppe ${ }^{1, *}$, Alberto Maria Gambelli ${ }^{2}$, Alberto Palliotti ${ }^{3}$, Andrea Nicolini $^{1,2}$, \\ and Federico Rossi ${ }^{1,2}$ \\ ${ }^{1}$ CIRIAF, Interuniversity Research Centre on Pollution and Environment "M. Felli”, Via G. Duranti \\ 67, 06125, Perugia, Italy \\ ${ }^{2}$ University of Perugia, Department of Engineering, Via G. Duranti 93, 06125, Perugia, Italy \\ ${ }^{3}$ University of Perugia, Department of Agriculture, Food and Environment, Borgo XX Giugno 74, \\ 06121 Perugia, Italy
}

\begin{abstract}
An innovative method against late frosts in vineyard has been developed at CIRIAF, University of Perugia. It consists of an organic coating made by cotton candy and straw to prevent the vine shoots freezing due to late frosts. This technique has already tested at lab-scale with very encouraging results. This paper aims to experiment the effect of the organic coating applied on young shoots of grapevine during artificially controlled frost events. A vine shoot covered by the organic coating was positioned in the climatic chamber and a thermocouple was rolled up to the vine shoot to control its temperature. Also, a vine shoot rolled up to the thermocouple and without organic coating was positioned inside the climatic chamber, used as a reference case. Results show that the temperature gap, gained with the adoption of the insulating layer was approximately in the range $0.2-3.2{ }^{\circ} \mathrm{C}$, but the organic coating was not enough to maintain temperature above the ice-point able to prevent the vine shoot freezing in lab. Even thought, the experimental application of the present technique allowed to detect some crucial challenges which need to be solved before to test this technology in real situations.
\end{abstract}

\section{Introduction}

The impact of climate change in the viticulture sector is well explained considering that warmer temperatures are associated with the earlier occurrence of the phenological grapevine phases [1-4]. In particular, Leolini et al. [3] suggested that budburst will occur 711 days on average earlier in Europe under the ongoing climate change. As consequences, an anticipated budburst may expose young shoots to an increased risk of late frosts, resulting in negative influences on grape composition and wine quality [5-7]. Thus, late frosts represent a serious threat to vineyard. In addition, considering the importance of the viticulture sector for the Italian economy $[8,9]$, many efforts are being made to counteract

\footnotetext{
* Corresponding author: alessia.digiuseppe@,crbnet.it
} 
the damages on vine shoots due to late frosts through innovative, sustainable and economic techniques instead of using already tested methods (e.g. both active and passive methods) [10-14].

In this framework, an innovative method against late frosts in vineyard has been developed at CIRIAF (Interuniversity Center on Pollution and Environment Mauro Felli) at University of Perugia [15]. It consists of an organic coating made by cotton candy and straw to prevent the vine shoots freezing due to late frosts. This technique has already tested at lab-scale with very encouraging results. A previous analysis in lab [15] showed that the organic coating was an excellent thermal insulator, able to reduce the energy consumption to maintain the temperature of an electric wire, used to simulate a vine shoots, up to $0^{\circ} \mathrm{C}$ and thus to prevent the late frost damages. Nonetheless, it was found that cotton candy degraded quickly in the presence of wind [15].

In a subsequent work [16], the straw was added to cotton candy showing to be able to slow down the degradation of cotton candy in windy condition and thus allowing it to perform its insulating function over a longer period. A preliminary cost analysis showed that the best solution to minimize overall costs consisted in creating an insulating layer made of $33.3 \mathrm{~g}$ of sugar per $\mathrm{m}^{2}$ and straw [16].

In the light of that framework, this paper aims to experiment the effect of the organic coating applied on young shoots of grapevine during artificially controlled frost events. A vine plant covered by the organic coating was positioned in the climatic chamber and a thermocouple was rolled up to the vine shoot to control its temperature. Also, a vine plant with the vine shoot rolled up to the thermocouple and without organic coating was positioned inside the climatic chamber, used as a reference case. This work represents the first stage in order to scale-up this technology and to test it in real situations.

\section{Materials and Methods}

This work represents a preliminary step in order to identify a material able to prevent the late frost damages in vineyard. This material must meet the following requirements: nontoxic, easy to use, cheap, and completely biodegradable. In this research, the possibility to apply an innovative method, already tested in lab $[15,16]$, in the real vine plants was investigated, allowing to detect some crucial challenges which need to be solved before to test this technology in real situations.

Two experiment tests were carried out with two pair of vine plants, characterized by similar phenological grapevine phases, in order to experiment the effect of the organic coating applied on young shoots of grapevine during artificially controlled frost events. The first pair of plants was small in size and was at the beginning of their vegetative cycle. The second one had started their cycle at least two weeks before the day of the experiment. The experimental tests were named Test 1 and Test 2 respectively.

The two pairs of vine plants tested are shown in Figure 1 below: 
a)
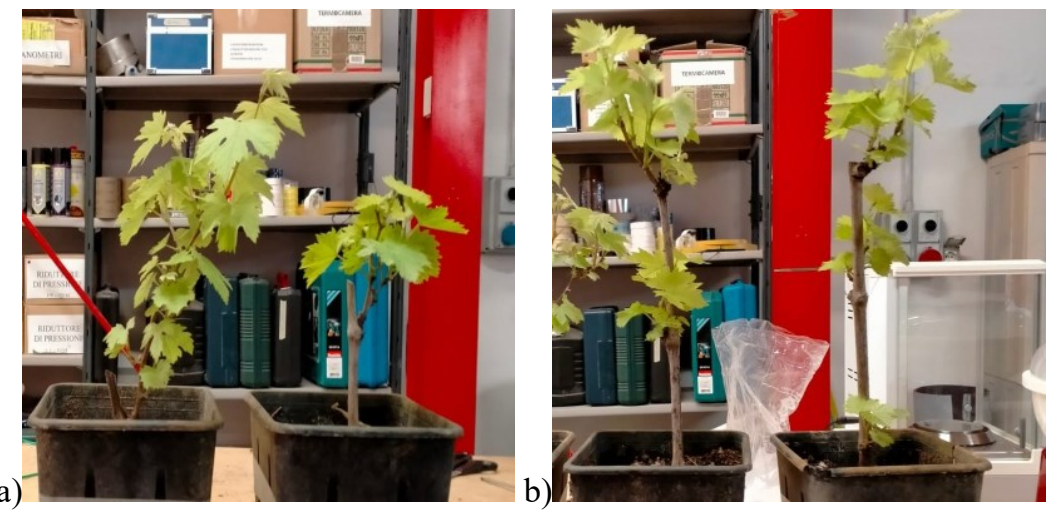

Fig. 1. The pair of smaller vine plants of the Test 1 (a) and the pair of taller vine plants of the Test 2 (b) before starting the experiment.

In both tests, for each pair of vine plants, one of them was covered by the insulating coating made of cotton candy and straw and compared to a control vine plant without insulating coating. In a previous work [16], the optimal sugar quantities, expressed as an optimum between insulating efficiency and costs, resulted equal to $33.3 \mathrm{~g}$ in case of a surface of $1 \mathrm{~m}^{2}$. Nonetheless, in these tests the quantity of sugar to be employed was equal to $20 \mathrm{~g}$ and $27 \mathrm{~g}$ in the Test 1 and 2, respectively, in order to cover the entire surface of plant with the cotton candy. According to the previous work [16], the tests were carried out by keeping the ratio between the grams of sugar and the grams of straw constant. In particular, $1 \mathrm{~g}$ of straw is used for every $5 \mathrm{~g}$ of sugar.

The tests were carried out inside the climatic chamber in which the temperature was set to $-6^{\circ} \mathrm{C}$ in order to simulate a late frost. The experimental facility consists of the following devices: (i) a climatic chamber (model MISA eptarefrigeration) with a control panel (model 202 expert) for refrigerated cells, (ii) a type $\mathrm{T}$ thermocouple, and (iii) a Lutron BTM4208SD data logger.

For each test, a vine plant was covered by the organic coating and a type $\mathrm{T}$ thermocouple was rolled up to the vine shoot to control its temperature. A reference case consisted by a vine plant without organic coating in which the vine shoot was rolled up to the thermocouple. In Figure 2 below, the plants of Test 2 after creating the insulating coating were shown.

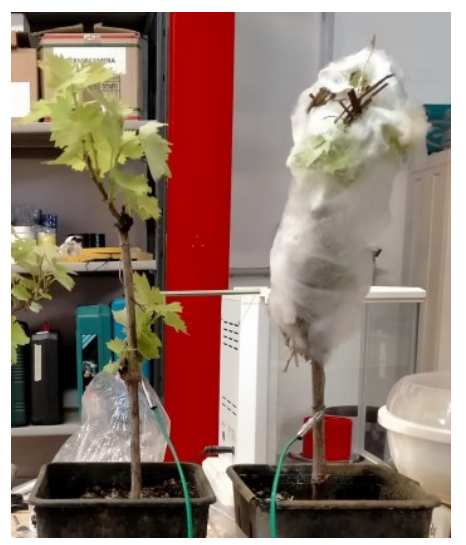

Fig. 2. Picture of the pair of plants of the Test 2 before beginning the experiment into the climatic chamber. 
Then the plants were positioned in the climatic chamber. The thermocouples were connected to a data logger in which the collected data are recorded with a two-minute timestep.

\section{Results and Discussion}

This section deals with two similar experiments, carried out according to what explained in section 2. Each experiment involved two plants. One of these plants was completely coated with candy cotton and straw, while the second was directly inserted in the refrigerating room.

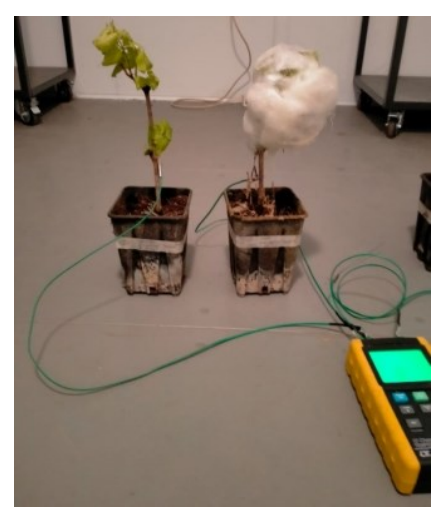

Fig. 3. Picture of the experimental set up before beginning Test 2: both plants were equipped with a thermocouple, used to detect temperature value and its variation over time in correspondence of a vine shoot; the plant on the right was completely coated with candy cotton and straw.

In Figure 3, the experimental set up is shown immediately before the activation of the cooling room. All plants were inserted closed to each other and temperature was continuously monitored along the whole test. The cooling device chosen for experiments was preferred to a climatic room, because of the necessity of carrying out experiments in absence of wind. During late frosts, the wind velocity is extremely low and can be neglected. Considering its strong influence on the coating maintenance over time, the present option was considered the most appropriate solution to perform an accurate description of late frosts in vineyards. The insulating material was entirely applied on the vine shoots, while the bole was kept free. Straw was directly mixed with candy cotton; then, a further layer was applied around the plant, to avoid leaks of straw before beginning the experiment. 
a)

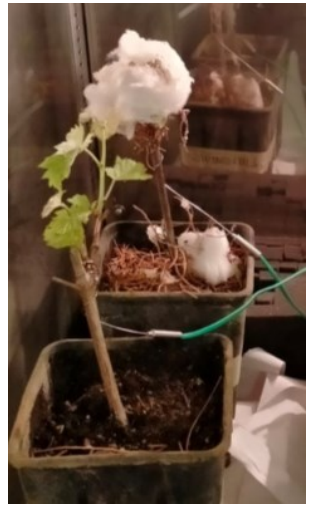

b)

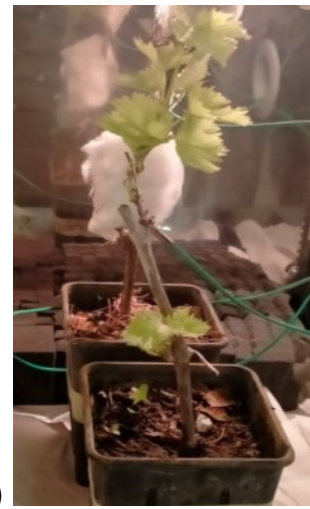

Fig. 4. Picture of plants Test 1 (a) and Test 2 (b) after 90 minutes from the beginning of experiments. The insulating layer has almost completely deteriorated.

Figure 4 represents a picture of plants after 90 minutes from the beginning of the experiment. The comparison between this figure and the previous clearly show the significant deterioration which interested the coating. Most of vine shoots were completely discovered and remained exposed to the external temperature without any protection.

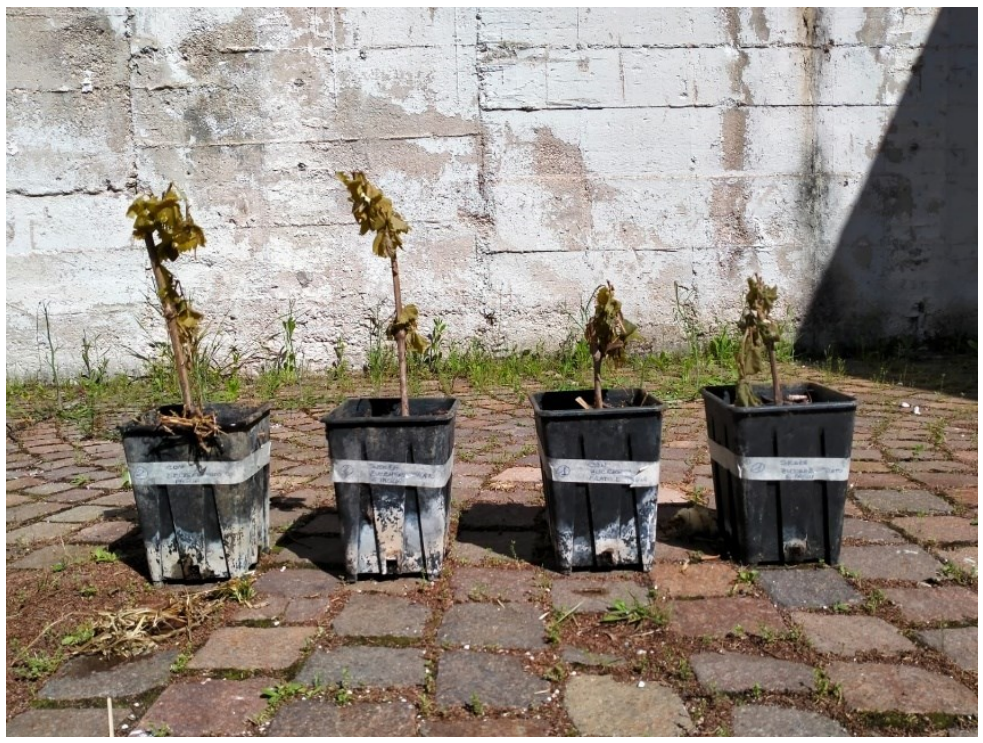

Fig. 5. Picture of plants as soon the test finished. The effect of temperature was visible in all of them; however, it was less severe in plant coated with candy cotton and straw.

In Figure 5, plants were shown immediately after being removed from the cooling room. Effects associated to the exposition to low temperatures are clearly visible and vine shoots resulted damaged in all cases. Temperature analysis over time, which is described below, together with the situation showed in Figure 4, explained the reason of it. After a certain time period, which was equal to $60-90$ minutes, the insulating efficiency of the coating was enough weak to make the difference in temperature between plants negligible. In addition, the environmental temperature was fixed at $-6{ }^{\circ} \mathrm{C}$ and the coating was not able to move temperature above the ice-point; it simply maintained milder conditions (with its presence, temperature was significantly higher than the environmental value). 
The following two diagrams describe the temperature trend over time for both couples of plants.

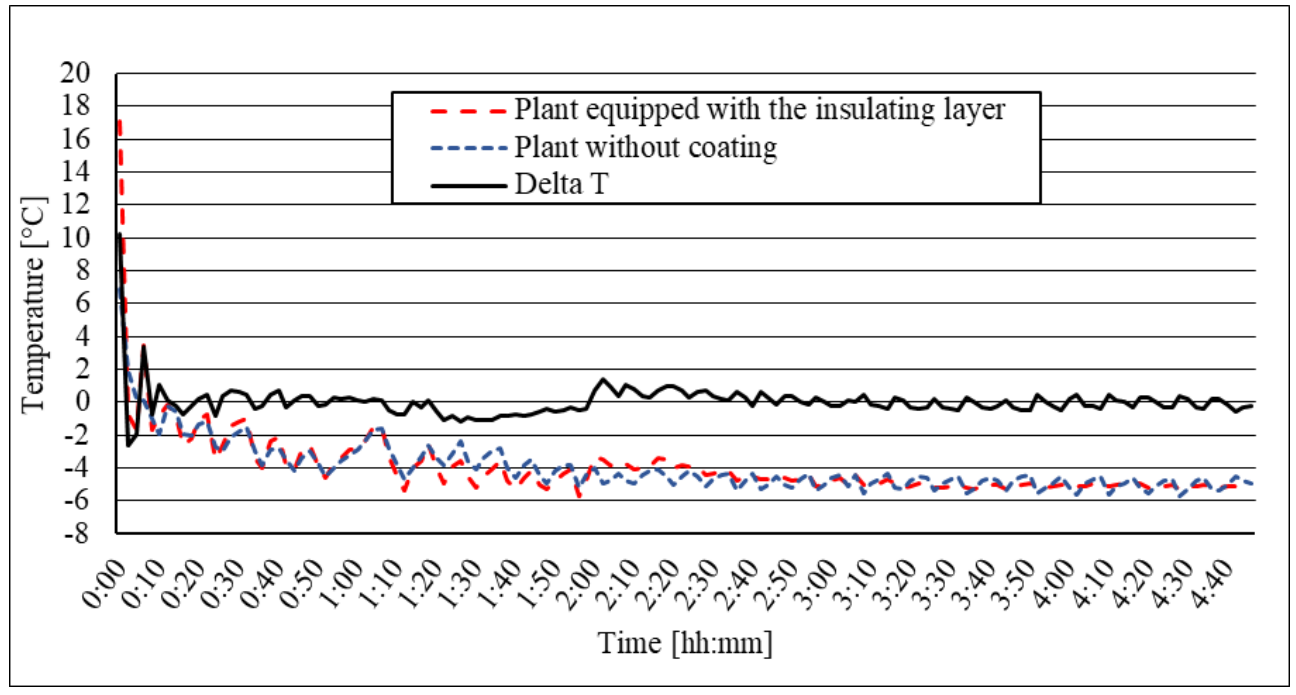

Fig. 6. Temperature trend over time for plants at the beginning of their vegetative cycle (Test 1).

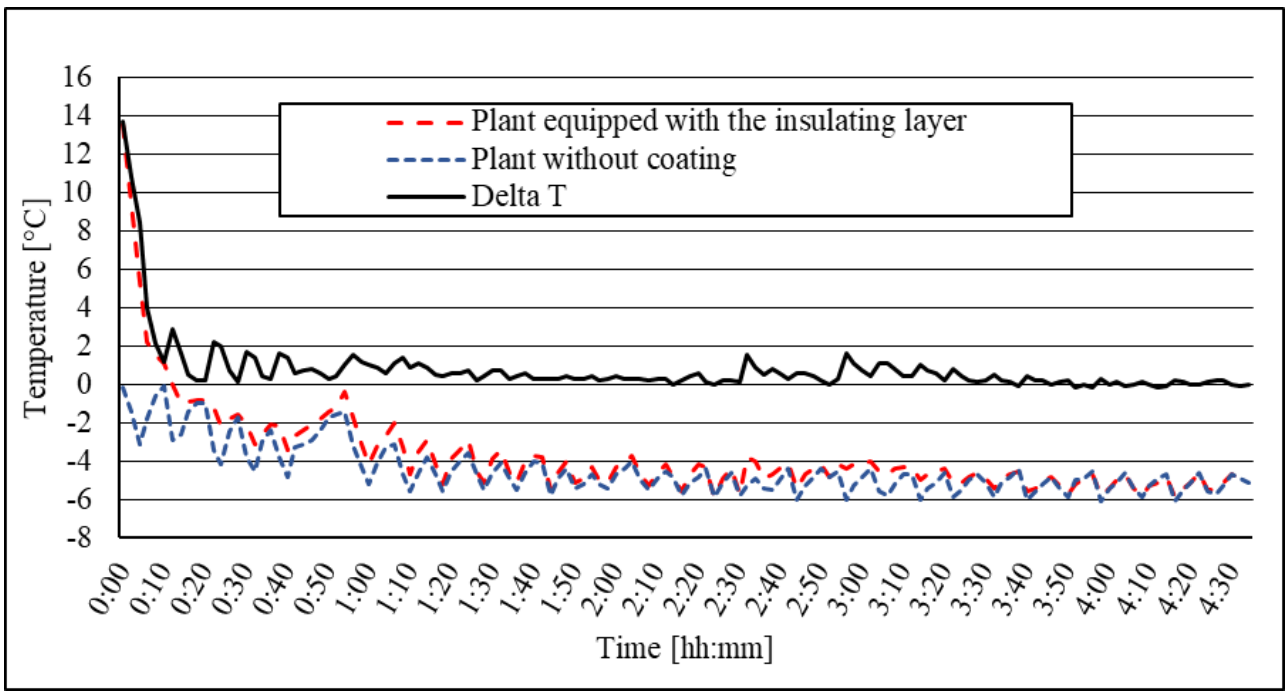

Fig. 7. Temperature trend over time for plants which started their vegetative cycle two weeks earlier than the date of experiment (Test 2).

In these diagrams, a red dotted line was used to show temperature of the plant equipped with the insulating layer, the blue dotted line is referred to plants without insulation and the continuous black line describes the difference between these two values. Figure 6 is associated to Test 1 and describes the situation observed with plants at the beginning of their vegetative cycle, while Figure 7 represents Test 2, where plants involved in the experiment started their vegetative cycle two weeks earlier than the date of experiment.

Both diagrams can be divided in three main phases. During the first minutes, the temperature of the coated plant is widely above the ice-point, while without candy cotton, it immediately moved from $0{ }^{\circ} \mathrm{C}$ to lower values. Such difference was not particularly meaningful, because it deepened from the initial temperature of candy cotton. During the 
following phase, temperature assumed a constant value on average in both cases, and the temperature gap, gained with the adoption of the insulating layer was approximately in the range $0.2-3.2^{\circ} \mathrm{C}$. This phase had a short time duration in both tests, if compared with the whole extension of the experiment $(\approx 5 \mathrm{~h})$. After approximately 60 minutes in Test 1 and 130 minutes in Test 2, the insulating efficiency of the coating became close to zero and the no difference in temperature was more registered between plants. In this situation, temperature moved below $-4{ }^{\circ} \mathrm{C}$ and vine shoots were damaged. A second addition of candy cotton was then performed. The quantity of raw material used in this phase was extremely lower than that involved to produce the first coating, in order to do not exceed excessively with the overall amount of sugar used. Any peak in temperature was registered: at the beginning of both experiments, plants were coated with candy cotton and straw before being inserted in the cooling room; in this latter case, plants were already inside and the coating layer lost all heat before being laid over plants. This led again to a visible difference in temperature between plants coated with sugar and plant without it (approximately $1-2^{\circ} \mathrm{C}$ ); however, it was not enough to maintain temperature above the ice-point. The effect associated to such second addition was less persistent than the previous one and, after some decades of minutes, the insulating effect disappeared completely.

\section{Conclusions}

An innovative method against late frosts in vineyard has been developed at CIRIAF, University of Perugia and tt consists of an organic coating made by cotton candy and straw. This technique has already tested at lab-scale with very encouraging results. In the light of above, this paper aims to experiment the effect of the organic coating applied on young shoots of grapevine during artificially controlled frost events. This brief experimental application of the present technique on vine plants allowed to detect some crucial challenges which need to be solved before going on with further steps (design of machinery for deposition of the coating on vineyards and so on):

The main findings are summarized in the following bullet points:

- As verified in previous works [15], the time duration of the coating represents a limiting factor for this application. It means that more resistant materials, having the same characteristics of candy cotton in terms of insulating efficiency, costs and simplicity of application, should be detected and tested.

- The temperature range in which such technique may effectively be a promising opportunity, must be better define. In absence of secondary sources of heat, an external temperature about six degrees lower than the ice-point may require the usage of a higher quantity of raw material per unit of surface than that defined in [16].

Further works will be proper focused on this aspect, and direct applications of the insulating layer on vine plants will be tested for several environment temperature, in the range from $0{ }^{\circ} \mathrm{C}$ to $-6{ }^{\circ} \mathrm{C}$. In addition, future efforts have been made in order to test the cotton candy with straw on vine shoots to protect them from late frost damages in real situations. The possible alteration and the effects on the organoleptic characteristics of the final products, by using these insulating organic materials in vineyards, will be considered and analysed.

\section{References}

1. IPPC. Summary for Policymakers. Climate Change 2013. The Physical Science Basis. Contribution of Working Group I to the Fifth Assessment Report on the 
Intergovernmental Panel on Climate Change; Cambridge University Press: Cambridge, UK, 2013.

2. E. Duchène, F. Huard, V. Dumas, C. Shneider, D. Merdinoglu, Clim. Res. 41, 193-204 (2010).

3. L. Leolini, M. Moriondo, G. Fila, S. Costafreda-Aumedes, R. Ferrise, M. Bindi, Field Crops Res. 222, 197-208 (2018).

4. C. Cuccia, B. Bois, Y. Richard, A.K. Parker, I.G. de Cortazar-Atauri, C. van Leeuwen, T. Castel, J. Int. Sci. Vigne. Vin. 48, 169-178 (2014).

5. R.M. de orduna, Food Res. Int. 43, 1844-1855 (2010).

6. G.V. Jones, M.A. White, O.R. Cooper, K. Storchmann, Clim. Chang. 73, 319-343 (2005).

7. A.D. Marta, D. Grifoni, M. Mancini, P. Storchi, G. Zipoli, S. Orlandini, J. Agric. Sci. 148, 657-666 (2010).

8. Scheda di settore - Vino. Available online: http://www.ismeamercati.it/report-analisiagroalimentare/schede-settore (accessed on 28 June 2021).

9. Focus Uve da Tavola. Available online: www.ismeamercati.it (accessed on 28 June 2021).

10. R.G. Evans, Proceedings of the ASEV 50th Anniversary Meeting, Seattle, WA, USA, 60-72 (2000).

11. E.bb. Poling, Hort. Science 43, 1652-1662 (2008).

12. R.G. Evans, Proceedings of the 104th Annual Meeting of the Washingtone State Horticultural Association, Yakima, WA, USA, 79-96 (2009).

13. F. Bonciarelli, U. Bonciarelli, Agronomia, 1st ed., 32-35 (2003).

14. Giving frost the cold shoulder. Available online: https://www.vineyardmagazine.co.uk/giving-frost-the-cold-shoulder/ (accessed on 28 June 2021).

15. A. Di Giuseppe, A.M. Gambelli, F. Rossi, A. Nicolini, N. Ceccarelli, A. Palliotti, Heat Transf. Res 51, 1625-1635 (2020).

16. A. Di Giuseppe, A.M. Gambelli, F. Rossi, A. Nicolini, N. Ceccarelli, A. Palliotti, Sustain. 12, 6279 (2020). 